\title{
Pakistan: Key Characters
}

\section{Governors-General}

Mohammad Ali Jinnah 1947-48

Khwaja Nazimuddin 1948-51

Ghulam Mohammad 1951-55

Iskander Mirza 1955-56

\section{Presidents}

Iskander Mirza 1956-58

General Mohammad Ayub Khan 1958-69

General Agha Mohammad Yahya Khan 1969-71

Zulfiqar Ali Bhutto 1971-73

Fazal Elahi Chaudhry 1973-78

General Mohammad Zia-ul-Haq 1978-88

Ghulam Ishaq Khan 1988-93

Farooq Khan Leghari 1993-97

Rafiq Tarar 1997-2001

General Pervez Musharraf 2001-8

Asif Ali Zardari 2008-present

Prime Ministers

Liaquat Ali Khan 1947-51

Khwaja Nazimuddin 1951-53

Mohammad Ali Bogra 1953-55

Chaudhry Mohammad Ali 1955-56

Husain Shaheed Suharwardy 1956-57 
Iftikhar I Chundrigar 1957 (Interim)

Feroz Khan Noon 1957-58

Zulfiqar Ali Bhutto 1973-77

Mohammad Khan Junejo 1985-88

Benazir Bhutto 1988-90

Ghulam Mustafa Jatoi 1990 (Interim)

Mian Mohammad Nawaz Sharif 1990-93

Balak Sher Mazari 1993 (Interim)

Moeenuddin Qureshi 1993 (Interim)

Benazir Bhutto 1993-96

Malik Meraj Khalid 1996-97 (Interim)

Mian Mohammad Nawaz Sharif 1997-99

Zafarullah Jamali 2002-4

Chaudhry Shujaat Hussain 2004 (Interim)

Shaukat Aziz 2004-7

Mian Mohamad Soomro (Interim)

Yusuf Raza Gilani 2008-2012

Raja Pervez Ashraf 2012-present

\section{Army Chiefs}

General Sir Frank Messervy 1947-48

General Sir Douglas Gracy 1948-51

General (Field Marshal) Mohammad Ayub Khan 1951-58

General Mohammad Musa Khan 1958-66

General Agha Mohammad Yahya Khan 1966-71

Lt. General Gul Hassan Khan 1971-72

General Tikka Khan 1972-76

General Mohammad Zia-ul-Haq 1976-88

General Mirza Aslam Beg 1988-91

General Asif Nawaz Janjua 1991-93

General Abdul Waheed 1993-96

General Jehangir Karamat 1996-98

General Pervez Musharraf 1998-2007

General Ashfaq Pervez Kayani 2007-present 


\section{Director Generals}

Lt. Gen. Ziauddin, Combat Development Directorate 1993-96

Lt. Gen. Zulfiqar Khan, Combat Development Directorate 1996-98

Lt. Gen. Khalid Kidwai Strategic Plans Division 1999-present

\section{Heads of Scientific Organizations}

Dr. Abdus Salam, Nobel Laureate

Chief Scientific Advisor to president of Pakistan, 1961-74

Chairman Pakistan Space and Upper Atmosphere Committee (SUPARCO), 1961-64

Founder International Center for Theoretical Physics, Trieste, Italy, 1964-93

\section{Pakistan Atomic Energy Commission (PAEC)}

Dr. Nazir Ahmad, April 1956-March 1960

Dr. Ishrat Husain Usmani, March 1960-March1972

Mr. Munir Ahmad Khan March, 1972-April 1991

Dr. Ishfaq Ahmad, April 1991-April 2001

Mr. Pervez Butt, April 2001-April 2006

Mr. Anwar Ali, 2006-9

Dr. Ansar Pervez, 2009-present

Khan Research Laboratory (KRL)

Dr. Abdul Qadeer Khan, July 1976-April 2001

Dr. Javed Mirza, April 2001-March 2006

Mr. Karim Ahmad, April 2006-present

National Engineering and Scientific Commission (NESCOM)

Dr. Samar Mubarkmand, January 2001-November 2007

Dr. Muhammad Irfan Burney, November 2007-present 
